Report Number DOE/SC0008028

\title{
Collaborative Research: Neutrinos \& Nucleosynthesis in Hot Dense Matter
}

Grant number $D E-S C 0008028$, June 1, 2012 - May 31, 2013

P. I. Sanjay Reddy, Team members: Gang Shen (Postdoc)

Institute for Nuclear Theory, University of Washington, Seattle, WA 98195

Final report submitted to the Nuclear Physics Division of the Office of Science of the Department of Energy

Report Covering the Period: June 1, 2012 - May 31, 2013 


\section{Executive Summary}

It is now firmly established that neutrinos, which are copiously produced in the hot and dense core of the supernova, play a role in the supernova explosion mechanism and in the synthesis of heavy elements through a phenomena known as r-process nucleosynthesis. They are also detectable in terrestrial neutrino experiments, and serve as a probe of the extreme environment and complex dynamics encountered in the supernova. The major goal of the UW research activity relevant to this project was to calculate the neutrino interaction rates in hot and dense matter of relevance to core collapse supernova. These serve as key input physics in large scale computer simulations of the supernova dynamics and nucleosynthesis being pursued at national laboratories here in the United States and by other groups in Europe and Japan. Our calculations show that neutrino production and scattering rate are altered by the nuclear interactions and that these modifications have important implications for nucleosynthesis and terrestrial neutrino detection.

The calculation of neutrino rates in dense matter are difficult because nucleons in the dense matter are strongly coupled. A neutrino interacts with several nucleons and the quantum interference between scattering off different nucleons depends on the nature of correlations between them in dense matter. To describe these correlations we used analytic methods based on mean field theory and hydrodynamics, and computational methods such as Quantum Monte Carlo. We found that due to nuclear effects neutrino production rates at relevant temperatures are enhanced, and that electron neutrinos are more easily absorbed than antielectron neutrinos in dense matter. The latter, was shown to favor synthesis of heavy neutronrich elements in the supernova. In what follows, I describe in some technical detail these findings.

\section{Accomplishments}

1. Spin-response of neutron and neutrino emissivity: Neutrinos in dense matter are primarily produced through fluctuations of the nucleon spin in the strongly coupled medium. The spectrum of such fluctuations is encoded in the dynamical spin response function. Until recently, all attempts to calculate the spin response of dense neutron and nuclear matter have relied on perturbation theory or other uncontrolled approximations. To assess the validity of these approximations we calculated the spin response of cold dense neutron matter using Quantum Monte Carlo (QMC) techniques which have proven to be the technique of choice for solving the many-body Schrödinger equation in nuclear systems. In an article published in Phys. Rev. C. 87, 025802 (2013), we showed that the frequency dependence of the longwavelength spin response is well constrained by sum-rules and the asymptotic behavior of the two-particle response at high frequency. The sum-rules are calculated using Auxiliary Field Diffusion Monte Carlo (AFDMC) technique and the high frequency two-particle response is calculated for several nucleon-nucleon potentials. At nuclear saturation density, the sumrules suggest that the strength of the spin response peaks at $\omega \simeq 40-60 \mathrm{MeV}$, decays rapidly for $\omega \geq 100 \mathrm{MeV}$, and has a sizable strength below $40 \mathrm{MeV}$. The form of the response function constructed using well motivated ansatze are shown in Fig. 1. We argued that this strength at relatively low energy could enhance neutrino production rates in dense neutron-rich matter at temperatures of relevance to core-collapse supernova. 


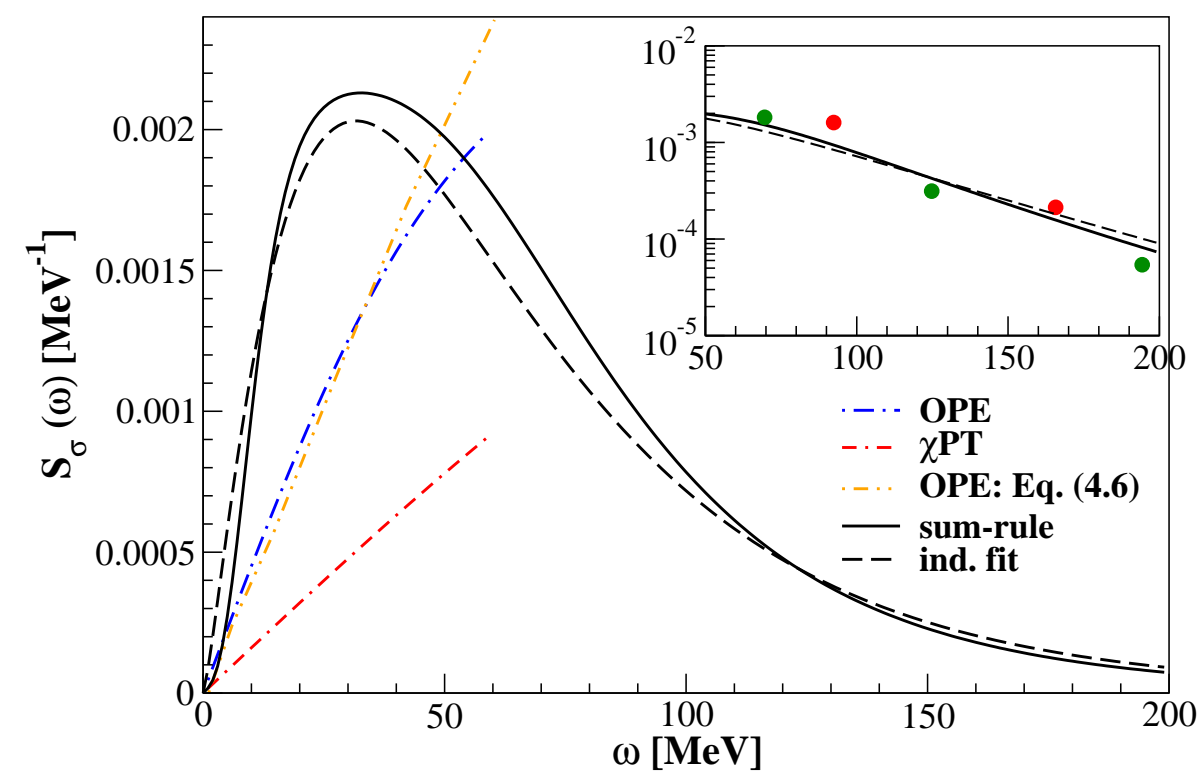

Figure 1: The spin response function $S_{\sigma}(q=0, \omega)$ of neutron matter at saturation density obtained by fitting to QMC sum-rules using two different ansatz are shown as the black solid and dashed curves. The inset compares the fits and the two-particle response at high energy obtained by confining two neutrons in a spherical cavity of radius $7 \mathrm{fm}$ (red) or $8 \mathrm{fm}$ (green). The linear, low-frequency forms predicted in earlier work based on the one-pion exchange $(\mathrm{OPE})$ and chiral perturbation theory $(\chi \mathrm{PT})$ are shown for comparison.

2. Charged current reactions in the neutrino sphere and nucleosynthesis: The spectrum of electron and anti-electron neutrinos emerging from the newly born neutron star called the proto-neutron star plays a crucial role in the synthesis of heavy elements in the supernova explosions. Previous work on neutrino emission from proto-neutron stars which employed full solutions of the Boltzmann equation showed that the average energies of emitted electron neutrinos and antineutrinos are closer to one another than predicted by older, more approximate work. This in turn implied that the neutrino driven wind is proton rich during its entire life, precluding $r$-process nucleosynthesis and the synthesis of Sr, Y, and Zr. This work relied on charged current neutrino interaction rates that are appropriate for a free nucleon gas. Here, it is shown in detail that the inclusion of the nucleon potential energies and collisional broadening of the response significantly alters this conclusion. Iso-vector interactions, which give rise to the nuclear symmetry energy, produce a difference between the neutron and proton single-particle energies $\Delta U=U_{n}-U_{p}$ and alter the kinematics of the charged current reactions. In neutron-rich matter, and for a given neutrino/antineutrino energy, the rate for $\nu_{e}+n \rightarrow e^{-}+p$ is enhanced while $\bar{\nu}_{e}+p \rightarrow n+e^{+}$is suppressed because the $Q$ value for these reactions is altered by $\pm \Delta U$, respectively. In the neutrino decoupling region, collisional broadening acts to enhance both $\nu_{e}$ and $\bar{\nu}_{e}$ cross-sections and 
RPA corrections decrease the $\nu_{e}$ cross-section and increase the $\bar{\nu}_{e}$ cross-section, but mean field shifts have a larger effect. Therefore, electron neutrinos decouple at lower temperature than when the nucleons are assumed to be free and have lower average energies. The spectrum of neutrinos predicted is shown in Fig. 2.

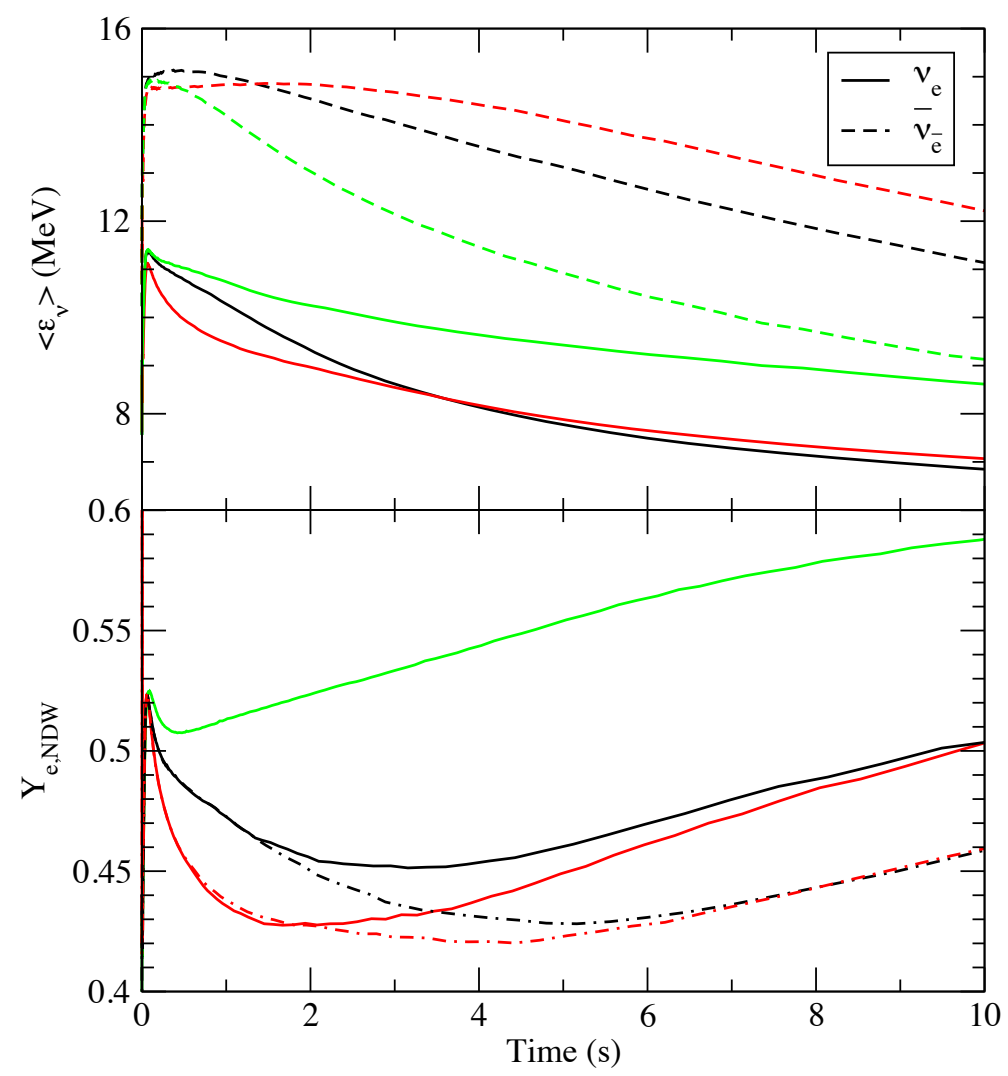

Figure 2: Spectra of electron neutrino and antineutrino as a function of time in three PNS cooling simulations. The solid lines are the average energies of the electron neutrinos and the dashed lines are for electron antineutrinos. The black lines correspond to a model which employed the a simple mean field equation of state with neutron matter properties that disagree with recent ab-initio calculations, and the red lines correspond to a model which employed an equation of state called IU-FSU which is in better agreement with ab-inition neutron matter calculations. The green lines are obtained in a model that neglects mean field effects on the neutrino opacities. Bottom panel: Predicted neutrino driven wind electron fraction as a function of time for the three models shown in the top panel (solid lines), as well as two models with the bremsstrahlung rate reduced by a factor of four (dot-dashed lines). The colors are the same as in the top panel.

The change is large enough to allow for a reasonable period of time when the neutrino driven wind is predicted to be neutron rich. It is also shown that the electron fraction in the wind is influenced by the nuclear symmetry energy.

3. Neutrino scattering from hydrodynamic modes When the neutrino wavelength is very large compared to the typical length scales in dense matter such as the nucleon mean 
free path and correlation lengths, theory of hydrodynamics can be used to describe neutrino scattering rates. In this work we calculate for the first time the scattering rates of low energy neutrinos in hot and dense neutron matter in the hydrodynamic regime. We find that the Brillouin peak associated with the sound mode, and the Rayleigh peak, associated with the thermal diffusion mode dominate the dynamic structure factor. These characteristic features are shown in Fig. 3. The total neutrino-matter scattering cross section is constrained by

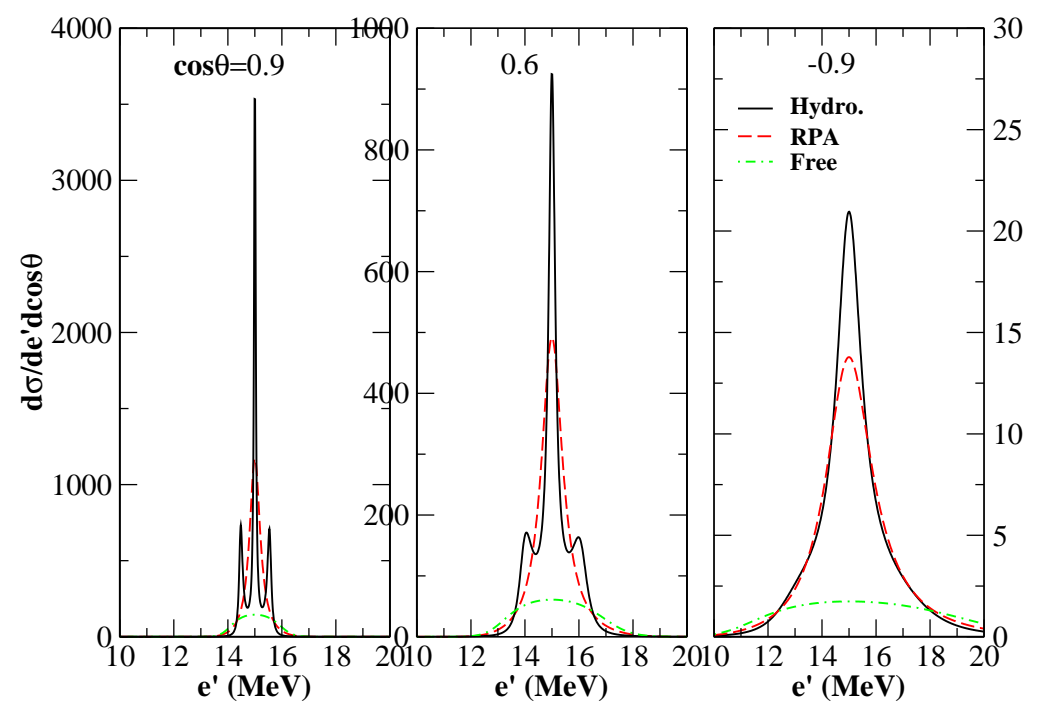

Figure 3: Differential cross section versus neutrino energy for various scattering angles, $\cos \theta$ $=0.9,0.6$, and -0.9 , obtained in the hydrodynamic approach, mean field theory and the free fermi gas approximation. Ambient conditions are neutron matter at $n=10^{-2} \mathrm{fm}^{-3}$ and $T=$ $5 \mathrm{MeV}$ and the incident neutrino energy was set to $3 T$.

the compressibility sum rule and determined by the equation of state of neutron matter. This study shows that low energy response can be characterized by a small number of well defined quantities: (i) sound speed, (ii)shear viscosity, and(iii) thermal conductivity, that capture the complex dynamics of the strongly interacting neutron gas. Our calculations have identified important differences between the response function obtained in the hydrodynamic limit and those commonly used in supernova simulations that are based on mean field theory or the random- phase-approximation (RPA) and the Fermi gas approximation. We are in the process of submitting the article for publication in Phys. Rev. C. where we discuss its implications for neutrino transport in supernova. 


\title{
Products
}

\author{
Journal Publications
}

1. G. Shen, S. Gandolfi, S. Reddy and J. Carlson, Spin Response and Neutrino Emissivity of Dense Neutron Matter, Phys. Rev. C 87, 025802 (2013) [arXiv:1205.6499 [nucl-th]].

2. L. F. Roberts, S. Reddy and G. Shen, Medium modification of the charged current neutrino opacity and its implications, Phys. Rev. C 86, 065803 (2012) [arXiv:1205.4066 [astro-ph.HE]].

Invited Talks (relating to this work)

1. Neutrino opacity in core collapse supernova, Invited talk by G. Shen at the inauguration symposium and workshop for Center for Nuclear Astrophysics, Shanghai Jiao Tong University, China, May 30, (2013).

2. Medium correction to neutrino interactions in core collapse supernova, seminar by G. Shen at Lawrence Berkeley National Laboratory, Berkeley, CA, USA, March 27, (2013).

3. Equation of State of dense matter and neutrino interactions around neutrino-sphere, invited talk by G. Shen at the Joint Institute for Nuclear Astrophysics (JINA) miniworkshop on EOS and weak interactions rates, Michigan State University, East Lansing, Michigan, November 11-16, (2012).

4. Nuclear Equation of State in Astrophysics: Effect of Nuclear Symmetry Energy invited talk by G. Shen at the Division of Nuclear Physics Fall Meet- ing, APS, Newport Beach, CA, October 26, (2012).

5. Phases of dense matter inside neutron stars and strategies to observe them, Physics Dept. Colloquium by S. Reddy at the University of North Carolina, March 18, (2013).

6. Dense matter in compact stars, Lectures (4) by S. Reddy at the 51st Winter school, Schladming, Austria, Feb. 23 -March 2, (2013).

\section{Collaborations initiated:}

To facilitate the incorporation of our improved calculations of neutrino rates into astrophysical simulations we are working with astrophysicists at Caltech (Christian Ott and Luke Roberts) and others including the supernova group at Oak Ridge National Laboratory (Raph Hix and his team), Princeton University (Adam Burrows) and Max Plank Institute in Garching, Germany (Thomas Janka and his team). Gang Shen is now developing a database of neutrino rates and fortran subroutines that can be used by the astrophysics community. 